\title{
A prospective controlled study of the association of Streptococcus bovis with colorectal carcinoma
}

\author{
M A Potter, N A Cunliffe, M Smith, R S Miles, A D Flapan, M G Dunlop
}

\begin{abstract}
Aim-To investigate the ability of Streptococcus bovis to colonise colorectal cancers.

Patients-19 patients with colorectal cancer and 23 controls without malignancy. Setting-University teaching hospital.

Methods-Prospective study comparing unselected patients with known colorectal cancer with age and sex matched controls. Carcinoma tissue from patients with colorectal cancer and normal colonic mucosa, stool, and blood from both patients and control subjects were cultured. Results-In contrast to published data, the faecal carriage rate was similar in cancer $(11 \%)$ and control groups $(13 \%)$. Conclusions-Faecal colonisation by Str bovis in colorectal cancer patients is lower than previously reported and does not differ significantly from controls.

(f Clin Pathol 1998;51:473-474)
\end{abstract}

Keywords: Streptococcus bovis; colorectal carcinoma

The reported high incidence of colorectal cancer in patients with Streptococcus bovis bacteraemia and endocarditis ${ }^{1-5}$ requires such patients to be rigorously investigated for the presence of a colonic tumour. ${ }^{6}$ Previous research indicates the faecal carriage of Str bovis is significantly higher in patients with colorectal cancer $(56 \%)$ than in controls $(11 \%),{ }^{3}$ but the underlying pathophysiological mechanisms are not understood. It has been speculated that undefined physical or biochemical factors in the gastrointestinal tract of patients with colonic carcinoma may encourage Str bovis faecal carriage, or alternatively that Str bovis may be locally carcinogenic. ${ }^{3}$ There are conflicting data on the importance of Str bovis common (c) antigen, which may be a specific marker of Str bovis bacteraemia, and may facilitate tumour attachment before translocation to the bloodstream. ${ }^{7}$ Preliminary work suggests that the detection of serum antibodies to Str bovis may provide a screening test to detect colon cancer. ${ }^{8}$ However, a recent small study found no evidence of Str bovis colonisation of six colorectal carcinomas. ${ }^{9}$ To further explore this association we investigated the ability of Str bovis to colonise colorectal cancer in a prospective, controlled study.

\section{Methods}

PATIENTS

Index cases with colorectal cancers were recruited prospectively without selection. Controls were selected from among patients having surgery for benign disease or diagnostic colonoscopy and were matched to the index cases for age and sex.

Based on published data, ${ }^{3}$ a power analysis indicated that 22 patients with colorectal cancer and 22 controls would give $80 \%$ power to detect a fivefold difference in Str bovis colonisation rates at the $\mathrm{p}<0.05$ level.

Local ethics committee approval and informed consent were obtained.

\section{CULTURES}

Stool and blood from patients with colorectal cancer were cultured preoperatively before any antibiotic administration. Colorectal carcinoma and adjacent macroscopically normal colonic mucosa were cultured immediately after surgical resection. All patients undergoing cancer resections received cefuroxime $750 \mathrm{mg}$ and metronidazole $500 \mathrm{mg}$ intravenously at anaesthetic induction.

Stool, blood, and normal mucosa were cultured from the control population. Control patients received no antibiotics during their hospital admission.

Faecal and fragmented tissue samples were separately plated onto bile aesculin azide agar (Gibco/BRL, Paisley, Scotland, UK) and incubated aerobically at $37^{\circ} \mathrm{C}$. Culture plates were examined after 24 and 48 hours for the presence of aesculin hydrolysing colonies. Ten aesculin hydrolysing colonies from each sample were plated individually onto $5 \%$ horse blood agar (Unipath, Bedford, UK) and incubated at $37^{\circ} \mathrm{C}$ overnight. $\alpha$ Haemolytic or nonhaemolytic streptococcal colony types were tested for Gram morphology and pyrolidonyl peptidase (PYR) activity (Murex Biotech, Dartford, UK). PYR negative organisms were subjected to Streptex rapid latex test (Murex Biotech) for streptococcal grouping. Lancefield group D or non-reactive streptococci were identified using the API 20 Strep system (Biomerieux, Basingstoke, UK). Streptococcus bovis isolates were confirmed and biotyped by the Streptococcal Reference Laboratory, Colindale, UK. Aerobic and anaerobic blood cultures (Roche Septichek, Becton Dickinson, Cowley, Oxford, UK) were incubated and regularly subcultured for two weeks before being categorised as negative.

\section{Results}

Stool samples were obtained from 24 patients and 27 controls. Unsatisfactory tissue samples reduced the study population to 19 patients (median age 73 years, range 44 to 87 ) and 23 controls (median age 58 years, range 15 to 80) (age difference not significant: $p=0.476$, 
Table 1 Carrier rates of Streptococcus bovis in clinical samples for 19 patients and 23 controls

\begin{tabular}{lll}
\hline & Patients & Controls \\
\hline Stool & $2(11)^{\star}$ & $3(13)^{\star}$ \\
Mucosa & $1(5.5)$ & $1(4)$ \\
Tumour & $2(11)$ & - \\
Blood culture & 0 & 0 \\
\hline
\end{tabular}

Values are $\mathrm{n}(\%) .{ }^{\star}$ Not significant $\left(\chi^{2}=0.052, \mathrm{p}=0.8,1 \mathrm{DF}\right.$ (Yates correction))

Mann-Whitney U test). The male to female ratio was 9:10 in the patient group and 11:12 in controls. Four patients underwent right hemicolectomy, one patient left hemicolectomy, 11 anterior resection, and three abdominoperineal resection. Seventeen patients had an adenocarcinoma on pathological examination (four Dukes stage A, eight Dukes B, and five Dukes $\mathrm{C}$ ), and two patients had large dysplastic adenomas.

In the control population 12 subjects underwent a diagnostic or surveillance colonoscopy which was normal, and 11 underwent haemorrhoidectomy or minor perianal surgery.

Str bovis was isolated from two patients with colorectal cancer and from three controls. In the study group one patient grew Str bovis in stool, mucosa, and tumour and one patient (with an adenoma) had positive cultures for stool and tumour. In the control population one subject had positive stool and mucosa cultures and two had a positive stool culture only (table 1). All positive cultures were biotype II. All cancer and control group preoperative blood cultures were negative.

\section{Discussion}

In this prospective study we found no significant difference in the preoperative faecal carriage rates between the study and control populations ( $11 \%$ versus $13 \%)$. This differs substantially from earlier work which found rates of $17-55 \%$, significantly greater than controls..$^{3-5}$ One patient in our cancer group with positive cultures for Str bovis later proved to have a large villous adenoma, indicating that adenomas are also susceptible to colonisation.

Str bovis can be confused with some viridans streptococci, for example Str salivarius, without rigorous physiological and serological tests, ${ }^{10}$ and this may account for the previous overestimate in Str bovis faecal carriage rate. ${ }^{3}$ Our bacteriological technique was thorough and employed elements from other published studies. ${ }^{39}$ Bile aesculin azide agar was used as previously reported, although only 10 colonies were selected in this study rather than $15 .^{3}$ The PYR screen was similar to that used by Norfleet and Mitchell, ${ }^{9}$ but the failure of some strains to react with latex grouping techniques ${ }^{10}$ means that such strains would have been missed in Norfleet's study.

One unavoidable confounding factor in this study concerns the culture of tumour and normal mucosa. Patients undergoing cancer resections received peroperative antibiotics as part of normal patient care, and delay in their administration would have been unethical. Although Str bovis is highly sensitive to $\beta$-lactam antibiotics, the tumour was devascularised shortly after the antibiotics were given, thereby limiting tumour exposure to antibiotics. Although we may have underestimated the prevalence of tumour colonisation, our methods appear robust because the two patients with positive stool cultures (taken before antibiotics) had tumour colonisation. In contrast only one of three control subjects with Str bovis faecal carriage had Str bovis cultured from the mucosa. These observations suggest that tumour colonisation is facilitated in some way.

Our data suggest that the incidence of Str bovis faecal colonisation in patients with known colorectal cancer is low and does not differ from control subjects without colorectal cancer. Str bovis carriage in the stool alone is not sufficient indication for colonic investigation. However, the observations of McMahon et al support the argument that investigation of patients with Str bovis bacteraemia is mandatory. ${ }^{1}$ It will be of interest to determine the natural history of Str bovis colonisation of the gut of those controls who are currently cancer-free.

This work was generously funded by The Royal Infirmary of Edinburgh Cancer Research Fund.

1 McMahon AJ, Auld CD, Dale BAS, et al. Streptococcus bovis septicaemia associated with uncomplicated colonic carcinoma. Br f Surg 1991;78:883-5.

2 Klein RS, Catalono MT, Edberg SC, et al. Streptococcus bovis septicaemia and carcinoma of the colon. Ann Intern Med 1979;91:560-2.

3 Klein RS, Recco RA, Catalano MT, et al. Association of Streptococcus bovis with carcinoma of the colon. $N$ Engl F Med 1977;297:800-2.

4 Honberg PZ, Gutschik E. Streptococcus bovis bacteraemia and its association with the alimentary tract neoplasm. Lancet 1987;i:163-4.

5 Leport C, Bure A, Leport J, et al. Incidence of colonic lesions in Streptococcus bovis and enterococcal endocardilis. Lancet $1987 ; \mathrm{i}: 748$.

6 Beeching NJ, Christmas TI, Ellis-Pegler RB, et al. Streptococcus bovis bacteraemia requires rigorous exclusion of colonic neoplasia and endocarditis. $Q \mathcal{F}$ Med 1985; 56:439-50.

7 Kaplan MH, Chamel H, Stephens A, et al. Humoral reactions in human endocarditis due to Streptococcus bovis: evidence for a common $\mathrm{S}$ bovis antigen. $\mathcal{F}$ Infect Dis 1983;148:266-74

8 Darjee R, Gibb AP. Serological investigation into the association between Streptococcus bovis and colonic cancer. $\mathcal{F}$ Clin Pathol 1993;46:1116-19.

9 Norfleet RG, Mitchell PD. Streptococcus bovis does not selectively colonise colorectal cancer and rectal polyps. $\mathcal{F}$ Clin Gastroenterol 1993;17:25-8.

10 Ruoff KL, Ferraro MJ, Holden J, et al. Identification of Streptococcus bovis and Streptococcus salivarius in clinical laboratories. F Clin Microbiol 1984;20:223-6. 